\title{
Model of exchange bias in a trilayer FM/AFM/ FM structure
}

Cite as: Low Temp. Phys. 41, 838 (2015); https://doi.org/10.1063/1.4934546

Published Online: 30 October 2015

M. L. Pankratova, and A. S. Kovalev
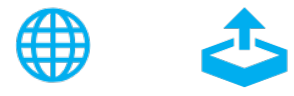

View Online

\section{ARTICLES YOU MAY BE INTERESTED IN}

Exchange bias and its propagation in ferromagnetic/antiferromagnetic/ferromagnetic trilayers Journal of Applied Physics 114, 153901 (2013); https://doi.org/10.1063/1.4824752

Propagation of exchange bias in CoFe / FeMn / CoFe trilayers

Applied Physics Letters 93, 152504 (2008); https://doi.org/10.1063/1.2999626

Exchange bias in NiFe / FeMn / NiFe trilayers

Journal of Applied Physics 96, 7428 (2004); https://doi.org/10.1063/1.1815048

LOW TEMPERATURE TECHNIQUES OPTICAL CAVI『V PHVSICS MITIGATING THERMAL \& VIBRATIONAL NOISE

\section{DOWNLOAD THE WHITE PAPER}




\title{
Model of exchange bias in a trilayer FM/AFM/FM structure
}

\author{
M. L. Pankratova ${ }^{\text {a) }}$ \\ B. Verkin Physics and Technology Institute for Low Temperatures, NAS Ukraine, 47 Lenin Ave., \\ Kharkov 61103, Ukraine
}

\section{A. S. Kovalev}

B. Verkin Physics and Technology Institute for Low Temperatures, NAS Ukraine, 47 Lenin Ave., Kharkov 61103, Ukraine, and V. N. Karazin Kharkov National University, 4 Svoboda Pl., Kharkov 61107, Ukraine (Submitted July 14, 2015)

Fiz. Nizk. Temp. 41, 1069-1076 (October 2015)

\begin{abstract}
A theoretical examination of the properties of the exchange bias phenomenon for sandwich FM/AFM/FM structures, which are actively studied in experiments. Magnetization dependences on the external magnetic field are obtained under the proposed discrete model of ferromagnetic layers of such multilayer structures. It is shown that the magnetization field dependences have a horizontal plateau, a splitting of the hysteresis loop, and asymmetry. We examine the influence of the interface defects on the exchange bias phenomenon and its features. The results are qualitatively consistent with experimental data. (C) 2015 AIP Publishing LLC.

[http://dx.doi.org/10.1063/1.4934546]
\end{abstract}

\section{Introduction}

The exchange bias (EB) phenomenon occurs in structures in which the ferromagnetic (FM) and antiferromagnetic (AFM) subsystems come into contact with one another. ${ }^{1-5}$ The effect is expressed as a shift in the magnetization dependence of the ferromagnetic film from the external magnetic field $M(H)$ along the axis of the field. The shift of the hysteresis loop can be accompanied by an asymmetric curve $M(H)$, the emergence of horizontal plateaus therein, sections with different plots and a splitting of the hysteresis loop. ${ }^{6,7}$ Regardless of the large number of experimental and theoretical studies, ${ }^{2-5}$ the causes behind the exchange bias are still not fully investigated. In theoretical studies EB has been associated with the appearance of domain walls in the $\mathrm{FM}^{4,8,9}$ or $\mathrm{AFM}^{10}$ part of the subsystem, and a roughness of the FM/AFM interface. ${ }^{11}$ In two-layer FM/AFM systems it is difficult to distinguish between the influence of the FM and AFM subsystem and their interfaces on the EB phenomenon and its properties. ${ }^{12}$ However, despite the lack of both theoretical and experimental research, the EB phenomenon is already used in technological applications. ${ }^{2}$ It is not the exchange bias itself that is important for such systems; in fact on average, it can be zero. What is important is the occurrence of various field dependences of magnetization for different directions of the external field, which provides for the possibility of controlling technical devices using weak magnetic fields. Recent experiments in sandwich FM/ AFM/FM structures have shown that in such systems it is possible to distinguish between inputs of different magnetic interactions. $^{12}$ In this trilayer structure, the two ferromagnetic layers can compensate for their impact on the antiferromagnetic layer, even during a reorientation in the magnetic field. The AFM magnetic moments remain fixed, and they are not reoriented by the external field. ${ }^{12,13}$ Partial reorientation of the magnetic moments at the FM/AFM interface leads to the training effect, but the magnitude of the shift and the main features of the magnetization remain the same. $^{12}$ The magnetization curves obtained for such structures show a split of the hysteresis loop by a horizontal plateau. The position of the plateau is $M / M_{\text {saturation }}=0.8$ and is determined by the different FM layer thicknesses in the structure. ${ }^{12}$ Also the magnetization dependence on the external magnetic field shows sloping regions of the curve.

The goal of this study is to theoretically describe EB and to study the reasons behind the occurrence of the horizontal plateau and the asymmetry in the sandwich FM/AFM/FM systems. ${ }^{12,14}$ We studied the influence of the exchange interaction in the FM film layer and through the FM/AFM interface, interface defects, and the emergence of inhomogeneity in the magnetization of the FM film. The system is studied within the framework of a simple discrete classic Heisenberg model, which reduces to the static version of the scalar sinusoidal Klein-Gordon equation. The article material is summarized as follows. In the second section we examine the sandwich FM/AFM/FM with two ideal FM/AFM interfaces. The third part examines how defects in the FM/AFM interface affect the properties of the field dependence of magnetization in a three-layer FM/AFM/FM structure.

\section{Discrete Model of Three-layer FM/AFM/FM Systems with Ideal Interfaces Between Layers}

A layered FM/AFM/FM system is considered. The AFM interlayer is considered to be magnetically hard, i.e., at the magnetic fields that are less than the spin-flop transition, its magnetic structure is fixed during the entire magnetization reversal. We consider a case of uncompensated FM/AFM interfaces, i.e., layered AFM in which the average magnetic moment is not zero in the layers closest to the interface. At the same time, we distinguish those situations with an even and odd number of AFM layers, i.e., with a different or identical direction of magnetization at the FM/AFM interface, which influences the magnetization in the ferromagnetic layers. For most of this paper, we consider a case of an odd number of AFM layers. In this case the magnetization in the 


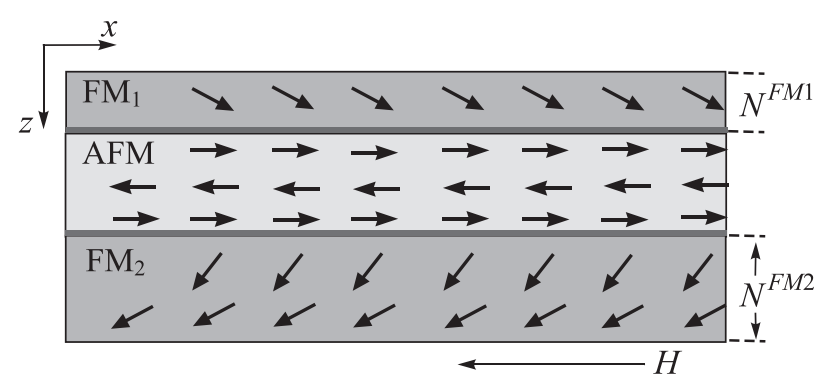

FIG. 1. A diagram of the discrete trilayer FM/AFM/FM system with ideal interfaces.

AFM layers closest to the interfaces runs in a parallel direction (Fig. 1). The question of an even number of AFM layers is examined at the end of the study. We take into account a strong easy-plane anisotropy in both FM films, which is determined, in part, by the magnetic dipole interaction. The orientation of the magnetic moments in the easy plane is determined by the angles $\varphi_{i}{ }^{F M j}$, where the superscript $j=$ 1,2 numbers two FM layers. In addition, we take into account the weak easy-axis anisotropy $\beta^{F M 1}, \beta^{F M 2}$ in the easy plane of the FM layer. The parameters of the magnetic anisotropy can bee differentiated in the first and second FM layers: $\beta^{F M 1} \neq \beta^{F M 2}$. Furthermore, we took into account the surface anisotropy of the interface, i.e., the different value of the anisotropy parameters for the atomic ferromagnetic layer closest to the FM/AFM interface, and at the free surface of the FM film: $\beta_{1}^{F M 1} \neq \beta_{2}^{F M 2}$. We examine FM layers of varying thickness $N^{F M 1} \neq N^{F M 2}$ ( $N$ is the number of atomic layers in the FM subsystem), so that each FM layer transition would be easily identifiable. The exchange interactions in the FM films are characterized by the parameters $J_{0}^{\mathrm{FM} 1}$, $J_{0}^{\mathrm{FM} 2}$. The diagram of the proposed model is shown in Fig. 1. We assume that the structure of the FM layers is homogeneous along the $x$-axis in the figure.

The energy of this system is determined by expression

$$
\begin{aligned}
E= & -J_{0}^{F M 1} \cos \varphi_{1}^{F M 1}-J_{0}^{F M 2} \cos \varphi_{1}^{F M 2}-J^{F M 1} \sum_{n=1}^{N^{F M 1}-1} \cos \left(\varphi_{n}^{F M 1}-\varphi_{n+1}^{F M 1}\right)-J^{F M 2} \sum_{n=1}^{N^{F M 2}-1} \cos \left(\varphi_{n}^{F M 2}-\varphi_{n+1}^{F M 2}\right) \\
& -J^{F M 1} \sum_{n=2}^{N^{F M 1}} \cos \left(\varphi_{n}^{F M 1}-\varphi_{n-1}^{F M 1}\right)-J^{F M 2} \sum_{n=2}^{N^{F M 2}} \cos \left(\varphi_{n}^{F M 1}-\varphi_{n-1}^{F M 1}\right) \\
& -H \sum_{n=1}^{N^{F M 1}} \cos \varphi_{n}^{F M 1}-H \sum_{n=1}^{N^{F M 2}} \cos \varphi_{n}^{F M 2}-\frac{\beta^{F M 1}}{2} \sum_{n=1}^{N^{F M 1}} \cos ^{2} \varphi_{n}^{F M 1}-\frac{\beta^{F M 2}}{2} \sum_{n=1}^{N^{F M 2}} \cos ^{2} \varphi_{n}^{F M 2}
\end{aligned}
$$

The superscripts $\mathrm{FM}_{1}$ and $\mathrm{FM}_{2}$ number the first and second ferromagnetic layer, whereas the subscripts indicate the number of the atomic ferromagnetic layer $1 \leq n \leq N^{F M j}$ in the ferromagnetic films, where index 1 corresponds to the layer that is closest to the corresponding interface. The respective static configurations of the ferromagnet's magnetic moments are given by equations $\partial E / \partial \varphi_{i}{ }^{F M j}=0$, and look like

$$
\begin{aligned}
& \left(H+J_{0}^{F M 1}\right) \sin \varphi_{1}^{F M 1}+J \sin \left(\varphi_{1}^{F M 1}-\varphi_{2}^{F M 1}\right)+\frac{\beta^{F M 1}}{2} \sin \left(2 \varphi_{1}^{F M 1}\right)=0 \\
& H \sin \varphi_{n}^{F M 1}+J^{F M 1} \sin \left(\varphi_{n}^{F M 1}-\varphi_{n-1}^{F M 1}\right)+J^{F M 1} \sin \left(\varphi_{n}^{F M 1}-\varphi_{n+1}^{F M 1}\right)+\frac{\beta^{F M 1}}{2} \sin \left(2 \varphi_{n}^{F M 1}\right)=0, \\
& H \sin \varphi_{N^{F M 1}}^{F M 1}+J^{F M 1} \sin \left(\varphi_{N^{F M 1}}^{F M 1}-\varphi_{N^{F M 1}-1}^{F M 1}\right)+\frac{\beta^{F M 1}}{2} \sin \left(2 \varphi_{N^{F M 1}}^{F M 1}\right)=0 \\
& \left(H+J_{0}^{F M 2}\right) \sin \varphi_{1}^{F M 2}+J^{F M 2} \sin \left(\varphi_{1}^{F M 2}-\varphi_{2}^{F M 2}\right)+\frac{\beta^{F M 2}}{2} \sin \left(2 \varphi_{1}^{F M 2}\right)=0 \\
& H \sin \varphi_{n}^{F M 2}+J^{F M 2} \sin \left(\varphi_{n}^{F M 2}-\varphi_{n-1}^{F M 2}\right)+J^{F M 2} \sin \left(\varphi_{n}^{F M 2}-\varphi_{n+1}^{F M 2}\right)+\frac{\beta^{F M 2}}{2} \sin \left(2 \varphi_{n}^{F M 2}\right)=0, \\
& H \sin \varphi_{N^{F M 2}}^{F M 2}+J^{F M 2} \sin \left(\varphi_{N^{F M 2}}^{F M 2}-\varphi_{N^{F M 2}-1}^{F M 2}\right)+\frac{\beta^{F M 2}}{2} \sin \left(2 \varphi_{N^{F M 2}}^{F M 2}\right)=0 .
\end{aligned}
$$

These equations allow for solutions that describe socalled collinear structures $\varphi_{i}{ }^{F M 1}=\varphi_{i}{ }^{F M 2}=0, \pi$. In these states the magnetization of both the FM films is parallel or antiparallel to the direction of magnetization of the external
AFM surface layers. We will examine a particular case, depicted in Fig. 1, when the magnetization of two surface AFM layers coincides. In addition, the given equations allow for the solution of two anticollinear homogeneous structures 
with antiparallel ordering of homogeneous magnetization in two FM layers $\varphi_{i}{ }^{F M 1}=0, \varphi_{i}{ }^{F M 2}=\pi$ (anticollinear phase $\mathrm{AK}_{1}$ with ... $\uparrow \mid \downarrow \downarrow \ldots$, where the arrows indicate the magnetization in the FM layers, and the vertical line corresponds to the antiferromagnet dividing them). More complex anticollinear structures are also possible, in which the magnetization direction in the FM layers can depend on the number $n$ and be opposite: $\varphi_{i}^{F M j}=0, \varphi_{i+1}^{F M j}=\pi$ (anticollinear phase $\mathrm{AK}_{2}$ with ... $\left.\uparrow \mid \downarrow \uparrow \ldots\right)$. Moreover, the number $n$ can denote not only the number of the atomic ferromagnetic layer, but also the thin weakly-coupled layers in this film (compartmental model). ${ }^{12}$ Solutions for even more complex magnetic structures (canted phases) are permitted, with $\varphi_{i}^{F M 1}, \varphi_{i}^{F M 2} \neq 0, \pi$, different from those that are fully remagnetized.
For fixed values of magnetic anisotropy and exchange interaction parameters in FM films, the nature of the magnetic structure of this "sandwich" and its dependence on the external magnetic field are determined by the magnitude of the magnetic exchange interaction through the FM/AFM interfaces. Therefore, there is value in constructing a phase diagram $\left(J_{0}, H\right)$ to describe the regions in which different types of magnetic ordering for the system would exist, as a function of the interaction between the FM and AFM subsystems. Lines separating the regions of collinear, anticollinear, and canted structures, are given by expressions below, for the particular case of FM layers with a thickness of $N^{F M 1}=1, N^{F M 2}=2$. The type of dependence magnetization has on the field changes with the parameter of the exchange interaction through the FM/AFM interface

$$
\begin{aligned}
& J_{0}^{F M 1, F M 2}=-\frac{H^{2}+2 H J^{F M 2}+2 H \beta^{F M 2}+2 J^{F M 2} \beta^{F M 2}+\left(\beta^{F M 2}\right)^{2}}{H+J^{F M 2}+\beta^{F M 2}}(\uparrow \uparrow), \\
& J_{0}^{F M 1, F M 2}=-H^{2}-\beta^{F M 1}, \\
& J_{0}^{F M 1, F M 2}=-\frac{H^{2}-2 H J^{F M 2}-2 H \beta^{F M 2}+2 J^{F M 2} \beta^{F M 2}+\left(\beta^{F M 2}\right)^{2}}{H-J^{F M 2}-\beta^{F M 2}}(\downarrow \downarrow), \\
& J_{0}^{F M 1, F M 2}=-H^{2}+\beta^{F M 1}, \quad \\
& J_{0}^{F M 1, F M 2}=-\frac{H^{2}+2 J^{F M 2} \beta^{F M 2}-\left(\beta^{F M 2}\right)^{2}}{H+J^{F M 2}-\beta^{F M 2}}(\uparrow \downarrow) .
\end{aligned}
$$

The corresponding dependences $J_{0}^{F M 1, F M 2}(H)$ are shown in Fig. 2, where the arrows denote the regions in which collinear, anticollinear, and canted structures exist, and the vertical line separates the vectors that characterize the magnetization of ferromagnets $\mathrm{FM}_{1}$ and $\mathrm{FM}_{2}$. The dashed lines correspond to the values of the exchange interaction through the interface, at which there is a magnetization transition in $\mathrm{FM}_{1}$, and solid lines show a magnetization transition in

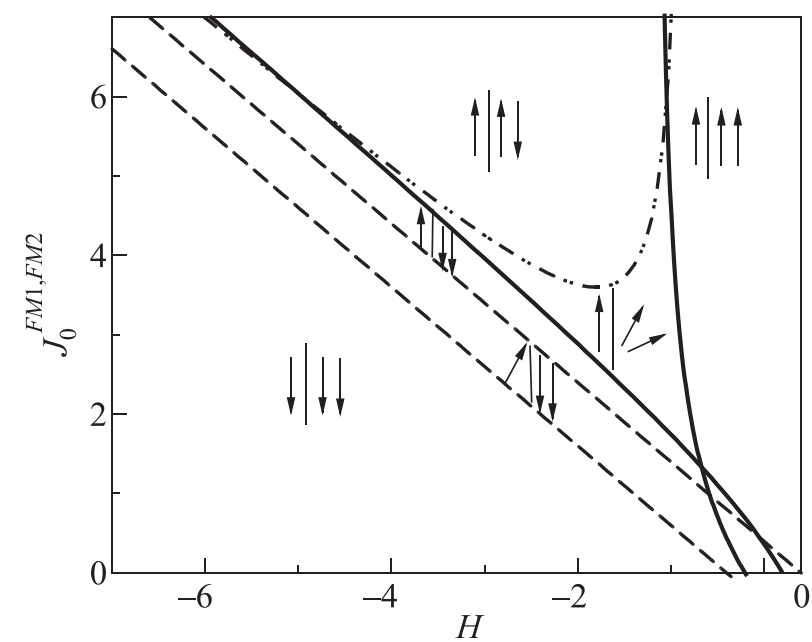

FIG. 2. Regions in which collinear, anticollinear, and canted structures exist in the FM part of the subsystem of an FM/AFM/FM sandwich, in the case of an ideal interface and varying parameters of magnetic anisotropy for the first and second FM layer $\beta^{F M 1}=0.4, \beta^{F M 2}=0.2, J^{F M 2}=1$.
$\mathrm{FM}_{2}$, the dash-dot line separates the region in which the horizontal plateau exists along the field dependence on magnetization.

The total magnetization for the given system is written as

$$
M=\sum_{n=1}^{N^{F M 1}} \cos \varphi_{n}^{F M 1}+\sum_{n=1}^{N^{F M 1}} \cos \varphi_{n}^{F M 2} .
$$

Its dependence on the external magnetic field was found numerically using a relaxation algorithm, described in better detail in Ref. 8. The obtained hysteresis loops are shown in Fig. 3, for different values of system parameters, in particular, for changes in the parameter of the exchange interaction through the FM/AFM interface, and changes in the parameter of magnetic anisotropy, as well as for a case of different parameters of exchange interaction through the interface for the first and second FM layer. All magnetization curves in this case are for a system with $N^{F M 1}=1, N^{F M 2}=2$. This ratio of ferromagnet layer thickness is selected in accordance with realistic experiments. Thus, for example, in Ref. 14 the thicknesses $\mathrm{FM}_{1} / \mathrm{AFM} / \mathrm{FM}_{2}$ are equal to $6 \mathrm{~nm} / 4 \mathrm{~nm} / 10 \mathrm{~nm}$.

In Fig. 3 we can see that in the proposed model it is possible to obtain all properties of the magnetic hysteresis, which were previously derived experimentally (Fig. 1 in Ref. 12, Fig. 1 in Ref. 14). We can conclude that the EB is associated with the formation of inhomogeneous states in the FM layer. The asymmetry $M(H)$ occurs due to varying 

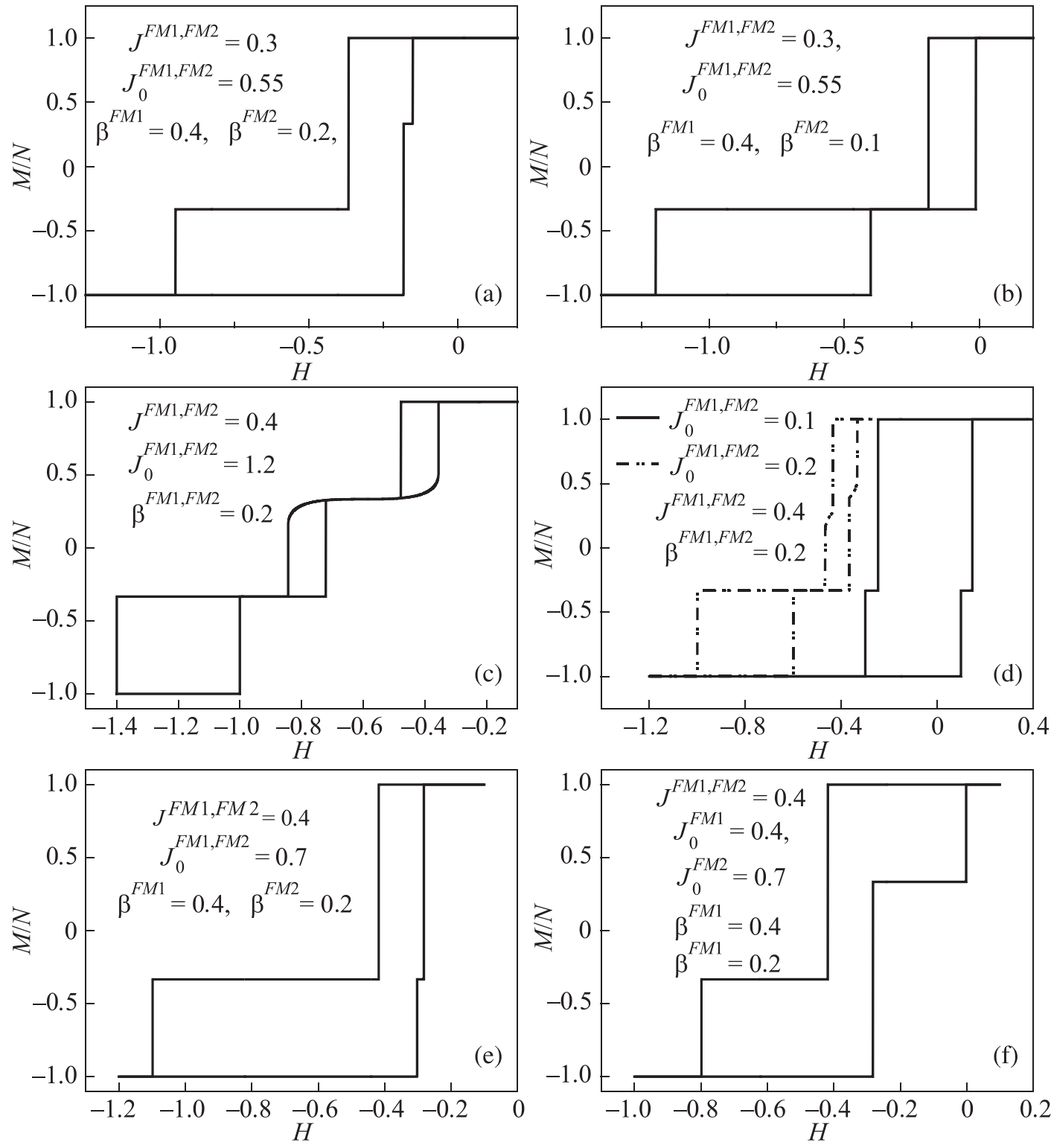

FIG. 3. Field dependences of magnetization for a sandwich FM/AFM/FM structure. Hysteresis loops are shown for different parameter values of exchange interaction and anisotropy, given ideal FM/AFM interfaces.

values of magnetic anisotropy in $\mathrm{FM}_{1}$ and $\mathrm{FM}_{2}$. The splitting of the hysteresis loop (Fig. 3(b)) can be caused by the transition of the first and second FM layer, and the position of the horizontal plateau along the magnetization dependence on the field is caused by the differing thicknesses of the FM films. Given an identical thickness of the first and second FM layer, there is no magnetization along the horizontal plateau: $M=0$.

\section{The Influence of Defects on the Properties of Magnetization Curves and EB}

In the previous section we obtained shifted magnetization field dependences with horizontal plateaus, hysteresis splitting, and canted regions along the curve. However, in real systems thanks to the roughness of the interface, only several percent of the magnetic moments interact through the FM/AFM interface. ${ }^{15}$ In our previous study, we proposed a model of point magnetic contacts at the FM/AFM interface, in order to explain the EB effect in a two-layer system with a rough interface. ${ }^{16}$ In this study we are examining a trilayer structure with one ideal and one rough FM/AFM interface (Fig. 4). In this case the magnetic exchange interaction occurs through the periodically arranged magnetic point contacts (MPC). The MPCs are periodically placed along the

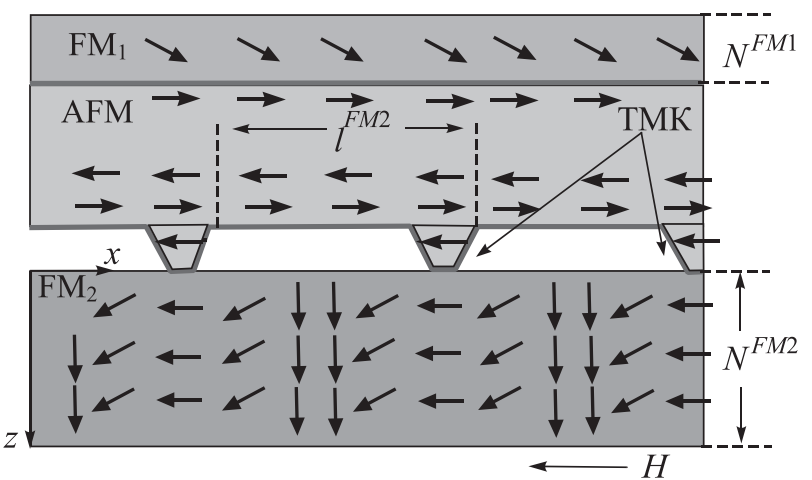

FIG. 4. Model of a sandwich FM/AFM/FM structure with one ideal and one inhomogeneous FM/AFM interface. 
FM/AFM interface surface along the $x$-axis, and the exchange interaction is facilitated only through the MPCs. Other magnetic moments through the interface do not interact. The magnetization of the ferromagnetic layers along the $z$-axis, perpendicular to the interface planes, is considered to be homogeneous, and domain walls can occur perpendicular to the interface in ferromagnetic subsystems.
As was the case in the prior example, a strong easy-plane anisotropy is taken into account, as well as an additional weak easy-plane anisotropy $\beta^{F M 1}, \beta^{F M 2}$. At the ideal interface the exchange interaction through the interface is equal to $J_{0}^{F M 1}$, and at the inhomogeneous interface the exchange parameter through MPC is equal to $J_{0}^{F M 2}$. The static configurations are characterized by the following equations:

$$
\begin{aligned}
& \left(H+J_{0}^{F M 1}\right) \sin \varphi_{1}^{F M 1}+J^{F M 1} \sin \left(\varphi_{1}^{F M 1}-\varphi_{2}^{F M 1}\right)+\frac{\beta^{F M 1}}{2} \sin \left(2 \varphi_{1}^{F M 1}\right)=0 \\
& H \sin \varphi_{n}^{F M 1}+J^{F M 1} \sin \left(\varphi_{n}^{F M 1}-\varphi_{n-1}^{F M 1}\right)+J^{F M 1} \sin \left(\varphi_{n}^{F M 1}-\varphi_{n+1}^{F M 1}\right)+\frac{\beta^{F M 1}}{2} \sin \left(2 \varphi_{n}^{F M 1}\right)=0, \\
& H \sin \varphi_{N^{F M 1}}^{F M 1}+J^{F M 1} \sin \left(\varphi_{N^{F M 1}}^{F M 1}-\varphi_{N^{F M 1}-1}^{F M 1}\right)+\frac{\beta^{F M 1}}{2} \sin \left(2 \varphi_{N^{F M 1}}^{F M 1}\right)=0 \\
& \left(H+J_{0}^{F M 2}\right) \sin \varphi_{1}^{F M 2}+J^{F M 2} \sin \left(\varphi_{1}^{F M 2}-\varphi_{2}^{F M 2}\right)+J^{F M 2} \sin \left(\varphi_{1}^{F M 2}-\varphi_{N^{F M 2}}^{F M 2}+\frac{\beta^{F M 2}}{2} \sin \left(2 \varphi_{1}^{F M 2}\right)=0,\right. \\
& H \sin \varphi_{n}^{F M 2}+J^{F M 2} \sin \left(\varphi_{n}^{F M 2}-\varphi_{n-1}^{F M 2}\right)+J^{F M 2} \sin \left(\varphi_{n}^{F M 2}-\varphi_{n+1}^{F M 2}\right)+\frac{\beta^{F M 2}}{2} \sin \left(2 \varphi_{n}^{F M 2}\right)=0, \\
& H \sin \varphi_{N^{F M 2}}^{F M 2}+J^{F M 2} \sin \left(\varphi_{N^{F M 2}}^{F M 2}-\varphi_{N^{F M 2}-1}^{F M 2}\right)+J^{F M 2} \sin \left(\varphi_{N^{F M 2}}^{F M 2}-\varphi_{1}^{F M 2}\right)+\frac{\beta^{F M 2}}{2} \sin \left(2 \varphi_{N^{F M 2}}^{F M 2}\right)=0 .
\end{aligned}
$$

The critical values of parameters $J_{0}^{F M 1, F M 2}(H)$ at which collinear structures occur, take the form of the following equations, when interface inhomogeneities are taken into account:

$$
\begin{aligned}
& J_{0}^{F M 1, F M 2}=-\frac{H^{2}+4 H J^{F M 2}+2 H \beta^{F M 2}+4 J^{F M 2} \beta^{F M 2}+\left(\beta^{F M 2}\right)^{2}}{H+2 J^{F M 2}+\beta^{F M 2}}(\uparrow \uparrow), \\
& J_{0}^{F M 1, F M 2}=-\frac{H^{2}-4 H J^{F M 2}-2 H \beta^{F M 2}+4 J^{F M 2} \beta^{F M 2}+\left(\beta^{F M 2}\right)^{2}}{H-2 J^{F M 2}-\beta^{F M 2}}(\downarrow \downarrow) .
\end{aligned}
$$

The given dependences are shown by dotted lines in Fig. 5, whereas solid lines represent curves $J_{0}^{F M 1, F M 2}(H)$ for a case of an ideal FM/AFM interface. We can see that for both types of interfaces, the magnetization curves will look qualitatively similar, but are at different values of system parameters (exchange interaction through the interface, and the magnetic anisotropy).

The obtained magnetization curves for the sandwich system with ideal interfaces, with inclusion of the interface inhomogeneity at one of the boundaries, are shown in Fig. 6. The hysteresis regions are marked by hatching. We can see that the hysteresis loop corresponding to the magnetization transition in $\mathrm{FM}_{1}$, retains its position and form. In this case the hatching indicates that the hysteresis loops for both of the trilayer systems overlap.

For such a system the field dependences of magnetization were obtained numerically at different MPC concentrations (Fig. 7). Hysteresis regions for the three different MPC concentrations are marked by hatching. We can see that by changing the degree of inhomogeneity at the interface, the horizontal plateau along the dependence shifts, and the hysteresis loops change shape. At the same time the hysteresis loop that corresponds to the magnetization transition in the $\mathrm{FM}_{1}$ layer, retains its position. Hysteresis regions for the three different degrees of inhomogeneity partially overlap.

The one-dimensional model being considered matches the linear contacts that are periodically placed along the

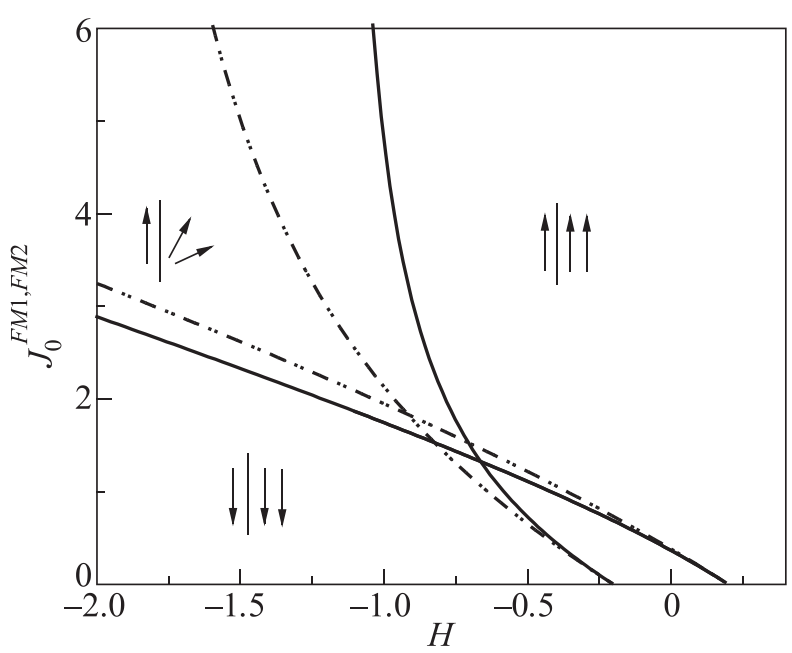

FIG. 5. Regions of collinear, anticollinear, and canted structures in the FM part of the subsystem in a sandwich structure with an ideal and rough FM/ AFM interface, and two ideal interfaces. 


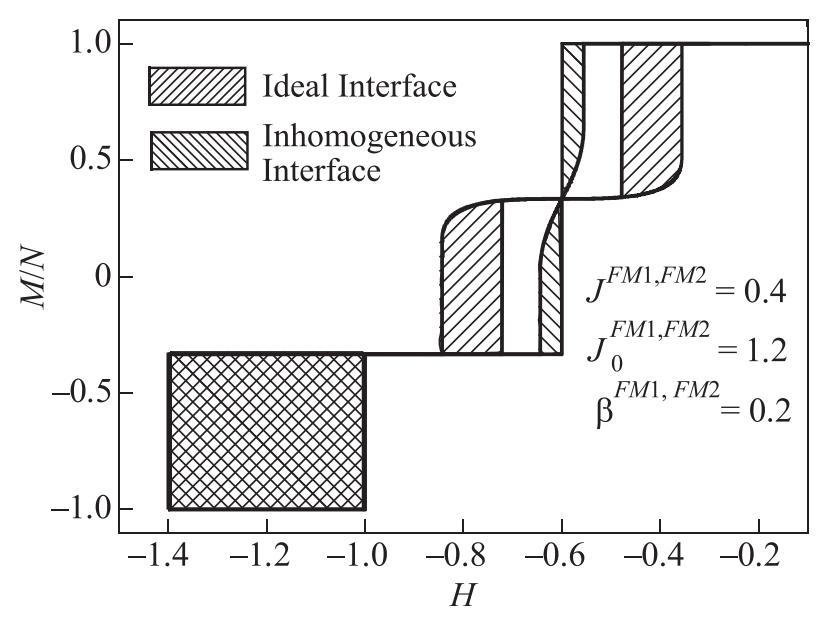

FIG. 6. The dependence of magnetization on the field $M(H)$, for a sandwich FM/AFM/FM structure, in the case of two ideal FM/AFM interfaces and an ideal and an inhomogeneous FM/AFM interface. The hysteresis regions are marked by hatching. In the bottom hysteresis loop that corresponds to the transition in $\mathrm{FM}_{1}$, the hysteresis loops for the different types of interfaces partially overlap.

$y$-axis. In reality, such contacts are randomly arranged along the FM/AFM interface surface. However, as shown in Ref. 6, the magnetization curves will look qualitatively similar, and will have the same shape for one-dimensional and two-dimensional periodic MPC systems, but with different system parameters.

The roughness of the interface can also be modeled by atomic steps at the interface. ${ }^{17}$ But in this case the properties of the hysteresis loops (horizontal plateau, canted regions of the curve and splitting of the hysteresis curve) are preserved. $^{17,18}$

It is necessary to remember that above we have examined a case of an odd number of layers in the AFM film (Fig. 1). In the case of an even number of layers in the AFM subsystem, the magnetization in the AFM layers closest to the interface at the first and second FM/AFM boundary, will be directed in opposition. As such, one of the FM films will be located in an additional field acting from the AFM, and

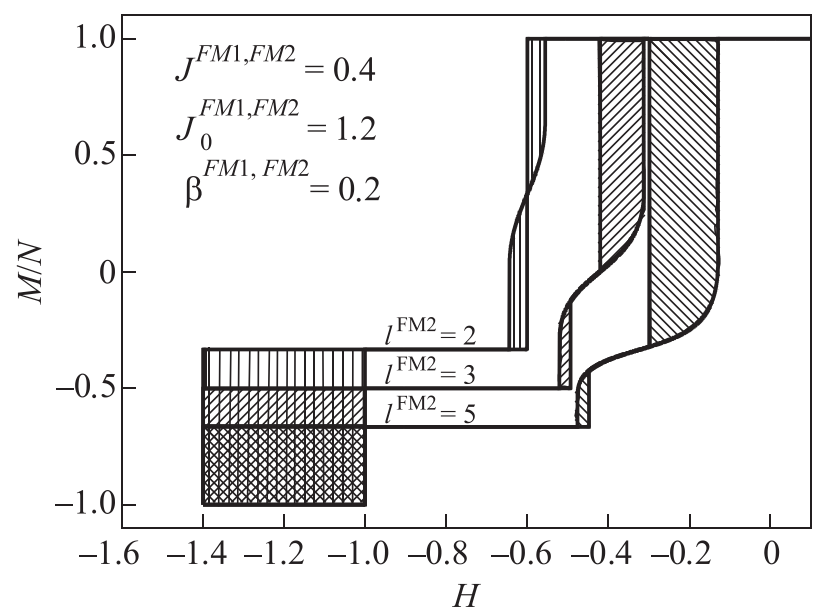

FIG. 7. Magnetization curves for a sandwich FM/AFM/FM system with one ideal and one inhomogeneous FM/AFM interface at varying degrees of inhomogeneity of the FM/AFM interface. Hysteresis regions are marked by hatching. In the bottom hysteresis loop that corresponds to the $\mathrm{FM}_{1}$ transition, the hysteresis loops for the different degrees of interface inhomogeneity, partially overlap.

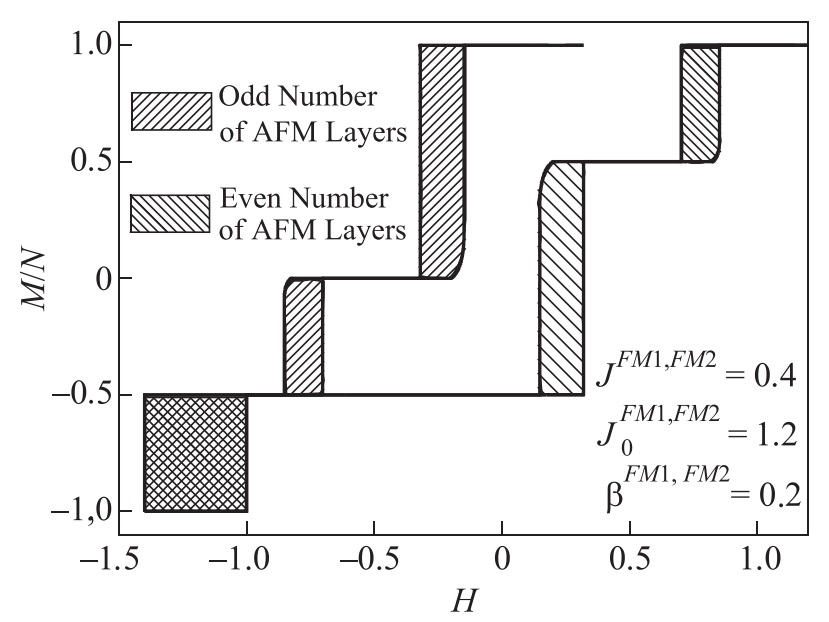

FIG. 8. Magnetization curves for a sandwich FM/AFM/FM system with ideal FM/AFM interfaces for an even and odd number of layers in the AFM. The hysteresis regions are marked by hatching. In the bottom hysteresis loop that corresponds to the $\mathrm{FM}_{1}$ transition, the hysteresis loops for both cases partially overlap.

directed in parallel to the external magnetic field. The magnetic moments of this FM film will start to rotate in the smaller magnetic field, and the hysteresis loop will shift into the positive field region. The corresponding hysteresis loops for AFM cases with an even and odd number of layers, are shown in Fig. 8. The magnetization curves are obtained for a case of two ideal FM/AFM interfaces at $N=4$.

In this study we examined a trilayer FM/AFM/FM system, in which the magnetic moments of the AFM remain fixed, whereas the FM subsystems do not interact. Therefore the actual results of this study generalize the results obtained earlier, for a two-layer FM/AFM model in Ref. 8 Furthermore the obtained magnetization dependences on the external magnetic field are actually a superposition of the magnetization field dependence for two ferromagnetic layers that are in contact with an AFM.

\section{Conclusion}

Within the framework of the discrete trilayer FM/AFM/ FM system, it is shown that the appearance of a horizontal plateau, asymmetry, and splitting of the hysteresis loop along the magnetization curve is associated with the formation of domain walls at the boundary of the FM part of the subsystem. We analyzed cases of an ideal and inhomogeneous FM/AFM interface. We found regions in which collinear, anticollinear, and canted structures exist. It is shown that the magnetization curves look qualitatively similar for both ideal and inhomogeneous interfaces. The obtained hysteresis field dependences of magnetization are in good qualitative agreement with the experimental results.

This study was partially supported by the Research Project NAS Ukraine No. 4/15-N.

a)Email: pankratova@ilt.kharkov.ua

${ }^{1}$ W. H. Meiklejohn and C. P. Bean, Phys. Rev. 102, 1413 (1956).

${ }^{2}$ J. Nogues and I. K. Schuller, J. Magn. Magn. Mater. 192, 203 (1999).

${ }^{3}$ A. E. Berkowitz and K. Takano, J. Magn. Magn. Mater. 200, 552 (1999).

${ }^{4}$ M. Kiwi, J. Magn. Magn. Mater. 234, 584 (2001).

${ }^{5}$ E. Fertman, S. Dolya, V. Desnenko, M. Kajňaková, and A. Feher, Fiz. Nizk. Temp. 40, 200 (2014) [Low Temp. Phys. 40, 156 (2014)]. 
${ }^{6}$ L. S. Uspenskaya, Phys. Status Solidi 52, 2274 (2010).

${ }^{7}$ C. Y. You, H. S. Goripati, T. Furubayashi, Y. K. Takahashi, and K. Hono, Appl. Phys. Lett. 93, 012501 (2008).

${ }^{8}$ A. G. Grechnev, A. S. Kovalev, and M. L. Pankratova, Fiz. Nizk. Temp. 38, 1184 (2012) [Low Temp. Phys. 38, 937 (2012)].

${ }^{9}$ A. G. Grechnev, A. S. Kovalev, and M. L. Pankratova, Fiz. Nizk. Temp. 39, 1361 (2013) [Low Temp. Phys. 39, 1060 (2013)].

${ }^{10}$ D. Mauri, H. C. Siegmann, P. S. Bagus, and E. Kay, J. Appl. Phys. 62, 3047 (1987).

${ }^{11}$ A. P. Malozemoff, Phys. Rev. B 35, 3679 (1987).

${ }^{12}$ A. N. Dobrynin and D. Givord, Phys. Rev. B 85, 014413 (2012).

${ }^{13}$ Y. Hu, F. Shi, N. Jia, Y. Liu, H. Wu, and An. Du, J. Appl. Phys. 114, 153901 (2013).
${ }^{14}$ G. Vallejo-Fernandez, L. E. Fernandez-Outon, and K. O’Grady, J. Appl. Phys. 103, 07C101 (2008).

${ }^{15}$ W. J. Antel, Jr., F. Perjeru, and G. R. Harp, Phys. Rev. Lett. 83, 1439 (1999).

${ }^{16}$ A. Kovalev and M. Pankratova, Superlattices Microstruct. 73, 275 (2014).

${ }^{17}$ A. S. Kovalev and M. L. Pankratova, Fiz. Nizk Temp. 40, 1267 (2014) [Low. Temp. Phys. 40, 990 (2014)].

${ }^{18}$ L. Anghinolfi, F. Bisio, M. Canepa, and L. Mattera, Phys. Rev. B 81, 224427 (2010).

Translated by A. Bronskaya 